J. Appl. Numer. Optim. 1 (2019), No. 1, pp. 77-90

Available online at http://jano.biemdas.com

https://doi.org/10.23952/jano.1.2019.1.07

\title{
WEAK SHARPNESS AND FINITE CONVERGENCE FOR MIXED VARIATIONAL INEQUALITIES
}

\author{
LUONG V. NGUYEN ${ }^{1,2}$, XIAOLONG QIN ${ }^{1, *}$ \\ ${ }^{1}$ Institute of Fundamental and Frontier Sciences, University of Electronic Science and Technology of China, Chengdu, China \\ ${ }^{2}$ Department of Natural Sciences, Hong Duc University, Thanh Hoa, Vietnam
}

\begin{abstract}
Weak sharp solutions of mixed variational inequalities are introduce and studied in Hilbert spaces. Several characterizations of weak sharpness of solutions of mixed variational inequalities without using gap functions are given. It is proved that sequences generated by an inexact proximal point algorithm terminate after a finite number of iterations for solutions of mixed variational inequalities provided that their solution sets are weakly sharp. Examples are also given to illustrate our results.

Keywords. Finite convergence; Mixed variational inequalities; Proximal point method; Weak sharpness.
\end{abstract}

2010 Mathematics Subject Classification. 49J40, 90C26.

\section{INTRODUCTION}

The notion of weak sharp minina for a convex optimization problem was introduced by Ferris [13]. It is a generalization of the notion of sharp minima due to Polyak [30] (or strongly unique local minima due to Cromme [12]) to include the case of a non-singleton solution set. This notion was later extensively established by many authors because of its impact on sensitivity analysis, error bounds and (finite) convergence analysis of a large number of optimization algorithms (see, e.g., [7, 8, 9, 10, 14, 18] and the references therein). Inspired by the notion of weak sharp minima for optimization problems, Patricksson [28] introduced the concept of weak sharp solutions for variational inequalities and studied the finite convergence of approximation algorithms for solving monotone variational inequalities under weak sharpness assumptions. Marcotte and Zhu [25] characterized weak sharpness of the solution set of a variational inequality in term of its dual gap function and studied finite convergence of sequences generated by some algorithms for solving variational inequalities under weak sharpness of the solution sets. Later, weak sharpness of solutions and its applications to the finite convergence property of methods for finding solutions of varitional inequalities have been investigated by many authors (see, e.g., $[3,17,23,24,26,27,32,33,34]$ and references therein). Some authors extended and established the concept of weak sharp solutions to general variational inequalities, e.g., set-valued variational inequalities [2, 31], variational-type inequalities [19], nonsmooth variational inequalities [4] and mixed variational inequalities [16].

\footnotetext{
* Corresponding author.

E-mail addresses: luonghdu@gmail.com (L.V. Nguyen), qxlxajh@163.com (X. Qin).

Received December 8, 2018; Accepted February 26, 2019.
} 
Mixed variational inequalities (MVIs) was initially considered by Lescarret [22] and Browder [6] in connection with it various applications in mathematical physics. Afterward, mixed variational inequality problems and their applications to mathematical physics, engineering, economics, operations research, electronics have extensively studied (see, e.g., [15, 20, 21, 29] and the references therein). It is known that MVIs generalize both variational inequality problems and minimization problems. Moreover, the first-order optimality conditions of a composite minimization problem are a particular case of mixed variational inequality problems. We refer the reader to [11] for more optimization problems which can be rewritten in the form of a mixed variational inequality.

In this paper, we will introduce the notion of weak sharpness for solutions of MVIs, establish several characterizations and study finite convergence properties under weak sharpness assumptions. The weak sharpness in this paper differs from the definition introduced by Huang and He in [16], in which the two authors introduced and characterized weak sharpness of solutions of MVIs in term of gap functions in the framework of Banach spaces. Our weak sharpness, which is a natural generalization, generalizes and unifies those of variational inequalities and of convex optimization problems.

We organize this paper as follows. In the next section, we recall some known definitions and results which will be used in the sequel. In Section 3, we first define the weak sharpness for solutions of MVIs and provide several interesting examples in which the solution sets of MVIs are weakly sharp. We then give several characterization results without using gap functions. These results generalize and improve known results in the literature. Finally, we study the finite convergence property of sequences generated by some algorithms for finding solutions of MVIs when the solution set is weakly sharp.

\section{PRELIMINARIES}

Let $H$ be a Hilbert space with inner product $\langle\cdot, \cdot\rangle$ and induced norm $\|\cdot\|$. Denote by $\mathbb{R}, \mathbb{R}_{+}$and $\mathbb{R}_{-}$ the set of real numbers, the set of nonnegative real numbers and the set of nonpositive real numbers, respectively. We denote by $\mathbb{B}$ the open unit ball in $H$. For a given nonempty subset $C$ of $H$, we denote by $\operatorname{int} C$ and $\operatorname{cl} C$ the interior and the closure of $C$, respectively. The polar cone $C^{\circ}$ of $C$ is defined by

$$
C^{\circ}:=\{y \in H:\langle y, x\rangle \leq 0, \quad \forall x \in C\} .
$$

For a given $x \in H$, the distance from $x$ to $C$ is defined by $d(x, C):=\inf _{y \in C}\|y-x\|$ and the projection of $x$ onto $C$ is defined by

$$
P_{C}(x):=\{y \in C:\|y-x\|=d(x, C)\} .
$$

It is known that $P_{C}(x)$ is a singleton set if $C$ is nonempty closed and convex. Note that the distance function $d(\cdot, C)$ is a nonexpansive mapping, i.e., $\|d(x, C)-d(y, C)\| \leq\|x-y\|$ for all $x, y \in H$.

Let $X$ be a nonempty closed convex subset of $H$. The tangent cone to $X$ at a point $x \in X$ is defined by

$$
T_{X}(x):=\operatorname{cl}\left(\bigcup_{\lambda>0} \frac{X-x}{\lambda}\right) .
$$

The normal cone to $X$ at $x \in X$ is defined by $N_{X}(x):=\left[T_{X}(x)\right]^{\circ}$, that is,

$$
N_{X}(x)=\{\zeta \in H:\langle\zeta, y-x\rangle \leq 0, \quad \forall y \in X\} .
$$

Let $f: H \rightarrow \mathbb{R} \cup\{+\infty\}$ be a proper, convex and lower semicontinuous function. The domain of $f$ is denoted by $\operatorname{dom}(f):=\{x \in H: f(x)<+\infty\}$. For $v \in H$ and $x \in \operatorname{dom}(f)$, the directional derivative of $f$ 
at $x$ in the direction $v$ is defined by

$$
f^{\prime}(x, v):=\lim _{t \rightarrow 0+} \frac{f(x+t v)-f(x)}{t} .
$$

The subdifferential of $f$ at $x \in \operatorname{dom}(f)$ is defined by

$$
\partial f(x):=\{\zeta \in H: f(y)-f(x) \geq\langle\zeta, y-x\rangle, \quad \forall y \in H\} .
$$

Definition 2.1. Let $F: H \rightarrow H$ be a mapping. Let $\theta: H \rightarrow \mathbb{R}$ be a function and let $K$ be a subset of $H$. The mapping $F$ is said to be

(i) monotone on $K$ if

$$
\langle F(x)-F(y), x-y\rangle \geq 0, \quad \forall x, y \in K
$$

(ii) pseudomonotone on $K$ if

$$
\langle F(x), y-x\rangle \geq 0 \Rightarrow\langle F(y), y-x\rangle \geq 0, \quad \forall x, y \in K ;
$$

(iii) $\theta$-pseudomonotone on $K$ if

$$
\langle F(x), y-x\rangle+\theta(y)-\theta(x) \geq 0 \Rightarrow\langle F(y), y-x\rangle+\theta(y)-\theta(x) \geq 0, \quad \forall x, y \in K .
$$

Remark 2.1. It is obvious that if $F$ is monotone on $K$, then it is pseudomotone and $\theta$-pseudomonotone on $K$. Pseudomononicity and $\theta$-pseudomonotonicity on $K$ of $F$ are independent.

From now on, we assume that $X$ is a closed convex subset of a Hilbert space $H, F: H \rightarrow H$ is a mapping and $\theta: H \rightarrow \mathbb{R}$ is a continuous convex function on $X$. We consider the following mixed variational inequality (MVI): Find $x^{*} \in X$ such that

$$
\left\langle F\left(x^{*}\right), x-x^{*}\right\rangle+\theta(x)-\theta\left(x^{*}\right) \geq 0, \quad \forall x \in X .
$$

If $\theta$ is constant on $X$, then (2.1) is reduced to the classical variational inequality (VI): Find $x^{*} \in X$ such that

$$
\left\langle F\left(x^{*}\right), x-x^{*}\right\rangle \geq 0, \quad \forall x \in X .
$$

Consider the following composite minimization problem (CMP):

$$
\min _{x \in X} \Phi(x):=f(x)+\theta(x),
$$

where $f: H \rightarrow \mathbb{R}$ is a differentiable convex function. One can verify that the first-order optimality conditions of (2.3) are a particular case of (2.1) with $F=\nabla f$. In particular, if $F \equiv 0$, then (2.1) is reduced to the following convex minimization problem (MP):

$$
\min _{x \in X} \theta(x) .
$$

For more optimization problems which can be rewritten in the form of mixed variational inequality (2.1), we refer the reader to [11] and the references therein.

We also consider the dual mixed variational inequality (DMVI): Find $x^{*} \in X$ such that

$$
\left\langle F(x), x-x^{*}\right\rangle+\theta(x)-\theta\left(x^{*}\right) \geq 0, \quad \forall x \in X .
$$

Denote by $X^{*}$ and $X_{*}$ the solution set of MVI (2.1) and of DMVI (2.5), respectively. We have the following properties concerning $X^{*}$ and $X_{*}$ which are easily to prove.

Proposition 2.1. The following assertions hold: 
(i) If $F$ is upper semicontinuous, then $X_{*} \subset X^{*}$.

(ii) If $F$ is $\theta$-pseudomonotone, then $X_{*}$ is closed, convex and $X^{*} \subset X_{*}$.

\section{MAIN RESULTS}

Burke and Ferris [7] introduced the notion of a weak sharp solution for a convex minimization problem (P): $\min _{x \in X} f(x)$, where $f: \mathbb{R}^{n} \rightarrow \mathbb{R}$ is a convex function and $X$ is a nonempty convex subset of $\mathbb{R}^{n}$. Recall that the solution set $X_{0}$ of the program (P) is weakly sharp if there exists a positive number $\alpha$ such that

$$
f(x) \geq f(\bar{x})+\alpha d\left(x, X_{0}\right)
$$

for all $\bar{x} \in X_{0}$ and $x \in X$. It was proved in [7] that if $f$ is a continuous differentiable convex function, $X$ and $X_{0}$ are nonempty closed convex, then $X_{0}$ is weakly sharp if and only if

$$
-\nabla f(\bar{x}) \in\left(\bigcap_{x \in X_{0}}\left[T_{X}(x) \cap N_{X_{0}}(x)\right]^{\circ}\right), \quad \forall \bar{x} \in X_{0} .
$$

In the case that $f$ is nondifferentiable, convex, $X_{0}$ and $X$ are nonempty closed convex, Burke and Ferris [7] proved that $X_{0}$ is weakly sharp if and only if there exists $\alpha>0$ such that

$$
\alpha \mathbb{B} \subset \partial f(\bar{x})+\left[T_{X}(\bar{x}) \cap N_{X_{0}}(\bar{x})\right]^{\circ}, \quad \forall \bar{x} \in X_{0} .
$$

Since variational inequality (2.2) lacks a "natural" objective function, Patriksson [28] (see also [25]) defined the weak sharpness of the solution set $X^{*}$ of (2.2). $X^{*}$ is said to be weakly sharp if

$$
-F\left(x^{*}\right) \in \operatorname{int}\left(\bigcap_{x \in X^{*}}\left[T_{X}(x) \cap N_{X^{*}}(x)\right]^{\circ}\right), \quad \forall x^{*} \in X^{*} .
$$

We are now in a position to define the weak sharpness of solutions of a mixed variational inequality problem. Since variational inequality problem (2.2) and minimization problem (2.4) are special cases of mixed variational inequality problem (2.1), it is natural to give a definition of weak sharpness of solutions of (2.1), which generalizes and unifies (3.2) and (3.3). From now on, we always assume that the solution set $X^{*}$ of (2.1) is nonempty closed and convex.

Definition 3.1. The solution set $X^{*}$ of the MVI (2.1) is said to be weakly sharp if, for any $x^{*} \in X^{*}$,

$$
-F\left(x^{*}\right) \in \operatorname{int}\left(\bigcap_{x \in X^{*}}\left(\partial \theta(x)+\left[T_{X}(x) \cap N_{X^{*}}(x)\right]^{\circ}\right)\right) .
$$

In other words, $X^{*}$ is weakly sharp if, for all $\bar{x} \in X^{*}$, there exists $\alpha>0$ such that

$$
\alpha \operatorname{cl} \mathbb{B} \subset F(\bar{x})+\bigcap_{x^{*} \in X^{*}}\left(\partial \theta\left(x^{*}\right)+\left[T_{X}\left(x^{*}\right) \cap N_{X^{*}}\left(x^{*}\right)\right]^{\circ}\right) .
$$

Remark 3.1. From the definition of weak sharpness of $X^{*}$, we can see that

(i) if $\theta$ is a constant function, then (3.4) is reduced to (3.3);

(ii) if $F \equiv 0$, then (3.4) is reduced to (3.2).

Remark 3.2. If $F$ is a constant on $X^{*}$, then the weak sharpness of $X^{*}$ is equivalent to the existence of a positive number $\alpha$ such that

$$
\alpha \operatorname{cl} \mathbb{B} \subset F\left(x^{*}\right)+\partial \theta\left(x^{*}\right)+\left[T_{X}\left(x^{*}\right) \cap N_{X^{*}}\left(x^{*}\right)\right]^{\circ} \text { for all } x^{*} \in X^{*} .
$$

We note that if (3.6) holds for some $\alpha>0$, then $X^{*}$ is weakly sharp in the sense of Huang and He [16]. 
We now give some examples in which the solution set of MVI is weakly sharp.

Example 3.1. Let $F: \mathbb{R}^{3} \rightarrow \mathbb{R}^{3}$ and $\theta: \mathbb{R}^{3} \rightarrow \mathbb{R}$ be defined by $F(x)=\left(x_{1}, x_{2}, 0\right)$ and $\theta(x)=x_{1}^{2}+x_{1}+$ $x_{2}^{2}+2 x_{2}+x_{3}^{2}+3 x_{3}$ for all $x=\left(x_{1}, x_{2}, x_{3}\right) \in \mathbb{R}^{3}$ and $X=\mathbb{R}_{+}^{3}$.

It is easy to check that $x^{*}=(0,0,0)$ is the unique solution of MVI (2.1), i.e., $X^{*}=\{(0,0,0)\}$. We can compute that $T_{X}\left(x^{*}\right)=\mathbb{R}_{+}^{3}$ and $N_{X^{*}}\left(x^{*}\right)=\mathbb{R}^{3}$. Thus,

$$
\left[T_{X}(x) \cap N_{X^{*}}(x)\right]^{\circ}=-\mathbb{R}_{+}^{3} .
$$

We have

$$
0=-F\left(x^{*}\right) \in \operatorname{int}\left(\bigcap_{x \in X^{*}}\left(\partial \theta\left(x^{*}\right)+\left[T_{X}(x) \cap N_{X^{*}}(x)\right]^{\circ}\right)\right)=\operatorname{int}\left((1,2,3)-\mathbb{R}_{+}^{3}\right),
$$

i.e., $X^{*}$ is weakly sharp.

Example 3.2. Let $H$ be a Hilbert space and let $X=\{x \in H:\|x\| \leq 5\}$. Let $F: H \rightarrow H$ be a nonnegative definite operator on $X$, i.e., $\langle F(x), x\rangle \geq 0$ for all $x \in X$, with $\|F(0)\|<1$, and let $\theta: H \rightarrow \mathbb{R}$ be defined by $\theta(x)=\|x\|$ for all $x \in H$. It is easy to verify that $x^{*}=0$ is the unique solution of MVI (2.1), i.e., $X^{*}=\{0\}$. We have $T_{X}\left(x^{*}\right)=H$ and $N_{X^{*}}\left(x^{*}\right)=H$. Thus

$$
\left[T_{X}\left(x^{*}\right) \cap N_{X^{*}}\left(x^{*}\right)\right]^{\circ}=\{0\} .
$$

Moreover, $\partial \theta\left(x^{*}\right)=\mathrm{clBB}$. One has

$$
-F\left(x^{*}\right)=-F(0) \in \mathbb{B}=\operatorname{int}\left(\bigcap_{x \in X^{*}}\left(\partial \theta\left(x^{*}\right)+\left[T_{X}(x) \cap N_{X^{*}}(x)\right]^{\circ}\right)\right) .
$$

Hence, $X^{*}$ is weakly sharp.

Example 3.3. Let $H=\mathbb{R}^{n}$ with the usual norm and let $X=\left\{x \in \mathbb{R}^{n}:\|x\| \leq 2\right\}$. Let $F: \mathbb{R}^{n} \rightarrow \mathbb{R}^{n}$ be a nonnegative definite operator on $X$, i.e., $\langle F(x), x\rangle \geq 0$ for all $x \in X$, with $F(0) \in(-1,1)^{n}$ and let $\theta: \mathbb{R}^{n} \rightarrow \mathbb{R}$ be defined by $\theta(x)=\left.|| x\right|_{1}:=\left|x_{1}\right|+\cdots+|x|_{n}$ for all $x=\left(x_{1}, \cdots, x_{n}\right) \in \mathbb{R}^{n}$. We see that $x^{*}=0$ is the unique solution of (2.1), that is, $X^{*}=\{0\}$. We have $T_{X}\left(x^{*}\right)=\mathbb{R}^{n}$ and $N_{X^{*}}\left(x^{*}\right)=\mathbb{R}^{n}$. Thus,

$$
\left[T_{X}\left(x^{*}\right) \cap N_{X^{*}}\left(x^{*}\right)\right]^{\circ}=\{0\} .
$$

We also have that $\partial \theta\left(x^{*}\right)=\partial \theta(0)=[-1,1]^{n}$ and

$$
-F\left(x^{*}\right)=-F(0) \in(-1,1)^{n}=\operatorname{int}\left(\bigcap_{x \in X^{*}}\left(\partial \theta\left(x^{*}\right)+\left[T_{X}(x) \cap N_{X^{*}}(x)\right]^{\circ}\right)\right) .
$$

Hence, $X^{*}$ is weakly sharp.

We next give an example in which the solution set $X^{*}$ is weakly sharp but $X^{*}$ is not a singleton.

Example 3.4. Let $X=[1,5] \times[0,2]$. Let $F: \mathbb{R}^{2} \rightarrow \mathbb{R}^{2}$ be defined by $F\left(x_{1}, x_{2}\right)=\left(0, x_{1}\right)$ for all $x=$ $\left(x_{1}, x_{2}\right) \in \mathbb{R}^{2}$ and let $\theta: \mathbb{R}^{2} \rightarrow \mathbb{R}$ be defined by $\theta\left(x_{1}, x_{2}\right)=x_{2}^{2}$ for all $x=\left(x_{1}, x_{2}\right) \in \mathbb{R}^{2}$. One can easily compute that the solution set of (2.1) is $X^{*}=[1,5] \times\{0\}$. For $x^{*} \in X^{*}$, we have $\partial \theta\left(x^{*}\right)=\{(0,0)\}$, $T_{X}\left(x^{*}\right)=\mathbb{R} \times \mathbb{R}_{+}$and $N_{X^{*}}\left(x^{*}\right)=\{0\} \times \mathbb{R}$. Hence

$$
T_{X}\left(x^{*}\right) \cap N_{X^{*}}\left(x^{*}\right)=\{0\} \times \mathbb{R}_{+} .
$$


For each $x^{*} \in X^{*}$, one has

$$
\partial \theta\left(x^{*}\right)+\left[T_{X}\left(x^{*}\right) \cap N_{X^{*}}\left(x^{*}\right)\right]^{\circ}=\mathbb{R} \times \mathbb{R}_{-} .
$$

Thus, for any $x^{*} \in X^{*}$, we have

$$
-F\left(x^{*}\right) \in\{0\} \times[-5,-1] \subset \operatorname{int}\left(\bigcap_{x \in X^{*}}\left(\partial \theta(x)+\left[T_{X}(x) \cap N_{X^{*}}(x)\right]^{\circ}\right)\right) .
$$

Hence, $X^{*}$ is weakly sharp.

We next give some characterizations of weak sharpness for solutions of MVIs.

Theorem 3.1. The following statements are equivalent:

(a) $X^{*}$ is weakly sharp.

(b) For each $\bar{x} \in X^{*}$, there exists $\alpha>0$ such that, for each $x \in X$,

$$
\alpha\left\|x-P_{X^{*}}(x)\right\|=\alpha d\left(x, X^{*}\right) \leq\left\langle F(\bar{x}), x-P_{X^{*}}(x)\right\rangle+\theta(x)-\theta\left(P_{X^{*}}(x)\right) .
$$

(c) For each $\bar{x} \in X^{*}$, there exists $\alpha>0$ such that, for any $x^{*} \in X^{*}$ and $v \in T_{X}\left(x^{*}\right) \cap N_{X^{*}}\left(x^{*}\right)$,

$$
\langle F(\bar{x}), v\rangle+\theta^{\prime}\left(x^{*}, v\right) \geq \alpha\|v\| .
$$

Proof. $(a) \Rightarrow(b)$ : Assume that $X^{*}$ is weakly sharp. Let $\bar{x} \in X^{*}$. Then, there exists $\alpha>0$ such that (3.5) holds. Thus,

$$
\alpha \operatorname{cl} \mathbb{B} \subset F(\bar{x})+\partial \theta\left(x^{*}\right)+\left[T_{X}\left(x^{*}\right) \cap N_{X^{*}}\left(x^{*}\right)\right]^{\circ}, \quad \forall x^{*} \in X^{*} .
$$

Hence, for any $x^{*} \in X^{*}$ and $0 \neq v \in T_{X}\left(x^{*}\right) \cap N_{X^{*}}\left(x^{*}\right)$, there exists $\zeta^{*} \in \partial \theta\left(x^{*}\right)$ such that

$$
\alpha \frac{v}{\|v\|}-F(\bar{x})-\zeta^{*} \in\left[T_{X}\left(x^{*}\right) \cap N_{X^{*}}\left(x^{*}\right)\right]^{\circ} .
$$

Hence

$$
\left\langle\alpha \frac{v}{\|v\|}-F(\bar{x})-\zeta^{*}, v\right\rangle \leq 0
$$

which implies that

$$
\alpha\|v\| \leq\left\langle F(\bar{x})+\zeta^{*}, v\right\rangle .
$$

The latter inequality also holds for $v=0$. Let $x \in X$. Since $X^{*}$ is closed convex, one finds that $P_{X^{*}}(x)$ is singleton and $\left\|x-P_{X^{*}}(x)\right\|=d\left(x, X^{*}\right)$ and

$$
x-P_{X^{*}}(x) \in T_{X}\left(P_{X^{*}}(x)\right) \cap N_{X^{*}}\left(P_{X^{*}}(x)\right) .
$$

Letting $v=x-P_{X^{*}}(x)$ and $x^{*}=P_{X^{*}}(x)$ in (3.9), we obtain

$$
\alpha\left\|x-x-P_{X^{*}}(x)\right\| \leq\left\langle F(\bar{x})+\zeta^{*}, x-P_{X^{*}}(x)\right\rangle .
$$

Since $\zeta^{*} \in \partial \theta\left(P_{X^{*}}(x)\right)$, one has

$$
\left\langle\zeta^{*}, x-P_{X^{*}}(x)\right\rangle \leq \theta(x)-\theta\left(P_{X^{*}}(x)\right) .
$$

Hence

$$
\alpha d\left(x, X^{*}\right)=\alpha\left\|x-P_{X^{*}}(x)\right\| \leq\left\langle F(\bar{x}), x-P_{X^{*}}(x)\right\rangle+\theta(x)-\theta\left(P_{X^{*}}(x)\right) .
$$


$(b) \Rightarrow(c)$ : The first part of proof follows some lines of the proof of [25, Theorem 4.1]. Fix $\bar{x} \in X^{*}$ and let $x^{*} \in X$. If $T_{X}\left(x^{*}\right) \cap N_{X^{*}}\left(x^{*}\right)=\{0\}$, then the proof is complete. Assume that $T_{X}\left(x^{*}\right) \cap N_{X^{*}}\left(x^{*}\right) \neq\{0\}$. Letting $v \in T_{X}\left(x^{*}\right) \cap N_{X^{*}}\left(x^{*}\right)$ be arbitrary with $v \neq 0$, we have $\langle v, v\rangle>0$ and

$$
\left\langle v, y^{*}-x^{*}\right\rangle \leq 0, \quad \text { for all } y^{*} \in X^{*} .
$$

These imply that

$$
X^{*} \subset H_{v}^{-}:=\left\{x \in H:\left\langle v, x-x^{*}\right\rangle \leq 0\right\} .
$$

Since $v \in T_{X}\left(x^{*}\right)$, for every positive sequence $\left\{t_{k}\right\}$ converging to 0 , there exists a sequence $\left\{v_{k}\right\}$ converging to $v$ and $x^{*}+t_{k} v_{k} \in X$ for all $k$. Then there exists $k_{0} \in \mathbb{N}$ such that $\left\langle v, v_{k}\right\rangle>0$ for all $k \geq k_{0}$. Thus

$$
\left.\left.\left\langle v, x^{*}+t_{k} v_{k}-x^{*}\right\rangle=t_{k}\right\rangle v, v_{k}\right\rangle>0, \quad \text { for all } k \geq k_{0},
$$

that is, $x^{*}+t_{k} v_{k} \notin H_{v}^{-}$for all $k \geq k_{0}$. By (3.10), we have, for all $k$, that

$$
d\left(x^{*}+t_{k} v_{k}, X^{*}\right) \geq d\left(x^{*}+t_{k} v_{k}, H_{v}^{-}\right) .
$$

For all $k \geq k_{0}$, the projection of $x^{*}+t_{k} v_{k}$ onto $H_{v}^{-}$belongs to

$$
H_{v}:=\left\{x \in H:\left\langle v, x-x^{*}\right\rangle=0\right\} .
$$

Fix $k \geq k_{0}$ and set $z_{k}:=P_{H_{v}}\left(x^{*}+t_{k} v_{k}\right)$. The vector $x^{*}+t_{k} v_{k}-z_{k}$ is a positive multiple of $v$, that is, there is a positive scalar $\gamma_{k}$ such that $x^{*}+t_{k} v_{k}-z_{k}=\gamma_{k} v$. Since $\left\langle v, z_{k}-x^{*}\right\rangle=0$, we have $\left\langle v, t_{k} v_{k}-\gamma_{k} v\right\rangle=0$. Thus

$$
\gamma_{k}=\frac{t_{k}\left\langle v_{k}, v\right\rangle}{\|v\|} \text {. }
$$

This gives

$$
z_{k}=x^{*}+t_{k} v_{k}-\frac{t_{k}\left\langle v_{k}, v\right\rangle}{\|v\|} v
$$

Then, for $k \geq k_{0}$, we have

$$
d\left(x^{*}+t_{k} v_{k}, H_{v}\right)=\left\|x^{*}+t_{k} v_{k}-z_{k}\right\|=\frac{t_{k}\left\langle v_{k}, v\right\rangle}{\|v\|} .
$$

This, together with (3.11), yields

$$
d\left(x^{*}+t_{k} v_{k}, X^{*}\right) \geq \frac{t_{k}\left\langle v_{k}, v\right\rangle}{\|v\|}, \quad \forall k \geq k_{0}
$$

Using the nonexpansiveness of the distance function, we have, for all $k \geq k_{0}$, that

$$
\begin{aligned}
d\left(x^{*}+t_{k} v, X^{*}\right) & \geq d\left(x^{*}+t_{k} v_{k}, X^{*}\right)-t_{k}\left\|v_{k}-v\right\| \\
& \geq \frac{t_{k}\left\langle v_{k}, v\right\rangle}{\|v\|}-t_{k}\left\|v-v_{k}\right\| .
\end{aligned}
$$

Since $t_{k}>0$ and $v \in N_{X^{*}}\left(x^{*}\right)$, one has $x^{*}=P_{X^{*}}\left(x^{*}+t_{k} v\right)$ for all $k$. By (3.7), for all $k \geq k_{0}$, one has

$$
\left\langle F(\bar{x}), x^{*}+t_{k} v-x^{*}\right\rangle+\theta\left(x^{*}+t_{k} v\right)-\theta\left(x^{*}\right) \geq \alpha \frac{t_{k}\left\langle v_{k}, v\right\rangle}{\|v\|}-\alpha t_{k}\left\|v_{k}-v\right\|,
$$

or, equivalently,

$$
\langle F(\bar{x}), v\rangle+\frac{\theta\left(x^{*}+t_{k} v\right)-\theta\left(x^{*}\right)}{t_{k}} \geq \alpha \frac{\left\langle v_{k}, v\right\rangle}{\|v\|}-\alpha\left\|v_{k}-v\right\| .
$$

Letting $k \rightarrow \infty$ and taking into account that $t_{k} \rightarrow 0, v_{k} \rightarrow v$, we get (3.8). 
$(c) \Rightarrow(a)$ : It follows from (3.8) that

$$
\sup \left\{\left\langle\zeta^{*}, v\right\rangle+\langle F(\bar{x}), v\rangle: \zeta^{*} \in \partial \theta\left(x^{*}\right)\right\} \geq \alpha\|v\|, \forall v \in T_{X}\left(x^{*}\right) \cap N_{X^{*}}\left(x^{*}\right) .
$$

We next show that, for all $v \in H$,

$$
\sup \left\{\left\langle\zeta^{*}, v\right\rangle+\langle F(\bar{x}), v\rangle: \zeta^{*} \in \partial \theta\left(x^{*}\right)+\left[T_{X}\left(x^{*}\right) \cap N_{X^{*}}\left(x^{*}\right)\right]^{\circ}\right\} \geq \alpha\|v\| .
$$

Suppose $v \notin T_{X}\left(x^{*}\right) \cap N_{X^{*}}\left(x^{*}\right)$. Then there exists $v^{*} \in\left[T_{X}\left(x^{*}\right) \cap N_{X^{*}}\left(x^{*}\right)\right]^{\circ}$ such that $\left\langle v^{*}, v\right\rangle>0$. Let $\zeta^{*} \in \partial \theta\left(x^{*}\right)$ and consider $\zeta^{*}+\lambda v^{*}+F(\bar{x})$ with $\lambda>0$. Since

$$
\left\langle\zeta^{*}+\lambda v^{*}, v\right\rangle+\langle F(\bar{x}), v\rangle \rightarrow \infty \text { as } \lambda \rightarrow \infty,
$$

the supremum in (3.14) is infinite. Thus (3.14) holds for all $v \notin T_{X}\left(x^{*}\right) \cap N_{X^{*}}\left(x^{*}\right)$. Assume now that $v \in T_{X}\left(x^{*}\right) \cap N_{X^{*}}\left(x^{*}\right)$. Since $0 \in\left[T_{X}\left(x^{*}\right) \cap N_{X^{*}}\left(x^{*}\right)\right]^{\circ}$, we have that

$$
\begin{aligned}
& \sup \left\{\left\langle\zeta^{*}, v\right\rangle+\langle F(\bar{x}), v\rangle: \zeta^{*} \in \partial \theta\left(x^{*}\right)\right\} \\
& \leq \sup \left\{\left\langle\zeta^{*}, v\right\rangle+\langle F(\bar{x}), v\rangle: \zeta^{*} \in \partial \theta\left(x^{*}\right)+\left[T_{X}\left(x^{*}\right) \cap N_{X^{*}}\left(x^{*}\right)\right]^{\circ}\right\} .
\end{aligned}
$$

This, together with (3.13), implies that (3.14) holds for all $v \in T_{X}\left(x^{*}\right) \cap N_{X^{*}}\left(x^{*}\right)$. Thus, (3.14) holds for all $v \in H$. Hence,

$$
\rho(v, \alpha \mathbb{B}) \leq \rho\left(v, F(\bar{x})+\partial \theta\left(x^{*}\right)+\left[T_{X}\left(x^{*}\right) \cap N_{X^{*}}\left(x^{*}\right)\right]^{\circ}\right),
$$

which implies from [1, Proposition 7] that

$$
\alpha \mathbb{B} \subset \operatorname{cl}\left(F(\bar{x})+\partial \theta\left(x^{*}\right)+\left[T_{X}\left(x^{*}\right) \cap N_{X^{*}}\left(x^{*}\right)\right]^{\circ}\right) .
$$

Therefore,

$$
\beta \mathbb{B} \subset F(\bar{x})+\partial \theta\left(x^{*}\right)+\left[T_{X}\left(x^{*}\right) \cap N_{X^{*}}\left(x^{*}\right)\right]^{\circ},
$$

with $0<\beta<\alpha$. This implies that

$$
-F(\bar{x}) \in \operatorname{int}\left(\bigcap_{x^{*} \in X^{*}}\left(\partial \theta\left(x^{*}\right)+\left[T_{X}\left(x^{*}\right) \cap N_{X^{*}}\left(x^{*}\right)\right]^{\circ}\right)\right) .
$$

So $X^{*}$ is weakly sharp. The proof is complete.

Corollary 3.1. Let $X^{*} \subset X_{*}$. For each $\bar{x} \in X^{*}$, set

$$
\alpha(\bar{x}):=\inf \left\{\frac{\langle F(\bar{x}), x-\bar{x}\rangle+\theta(x)-\theta(\bar{x})}{d\left(x, X^{*}\right)}: x \in X \backslash X^{*}\right\} .
$$

The following assertions are equivalent:

(i) $X^{*}$ is weakly sharp.

(ii) $\alpha(\bar{x})>0$ for each $\bar{x} \in X^{*}$.

Proof. Since $X^{*} \subset X_{*}$, we have

$$
\left\langle F(\bar{x}), \bar{x}-x^{*}\right\rangle+\theta(\bar{x})-\theta\left(x^{*}\right)=0
$$

for all $\bar{x}, x^{*} \in X^{*}$. Thus, for $\bar{x} \in X^{*}$ and $x \in X$, we have

$$
\left\langle F(\bar{x}), x-P_{X^{*}}(x)\right\rangle+\theta(x)-\theta\left(P_{X^{*}}(x)\right)=\langle F(\bar{x}), x-\bar{x}\rangle+\theta(x)-\theta(\bar{x}) .
$$


By Theorem 3.1, we have that $X^{*}$ is weakly sharp if and only if for each $\bar{x} \in X^{*}$ there exists $\alpha>0$ such that

$$
\alpha d\left(x, X^{*}\right) \leq\left\langle F(\bar{x}), x-P_{X^{*}}(x)\right\rangle+\theta(x)-\theta\left(P_{X^{*}}(x)\right) \text { for all } x \in X,
$$

which is equivalent to

$$
\alpha d\left(x, X^{*}\right) \leq\langle F(\bar{x}), x-\bar{x}\rangle+\theta(x)-\theta(\bar{x}) \quad \text { for all } x \in X .
$$

Hence, $\alpha(\bar{x})>0$ for each $\bar{x} \in X^{*}$. This completes the proof.

Example 3.5. We reconsider Example 3.4. One can also compute $X_{*}=[1,5] \times\{0\}=X^{*}$. For $\bar{x}=$ $\left(\bar{x}_{1}, \bar{x}_{2}\right) \in X^{*}$ and $x=\left(x_{1}, x_{2}\right) \in X \backslash X^{*}$, we have

$$
\alpha(\bar{x})=\inf \left\{\frac{\langle F(\bar{x}), x-\bar{x}\rangle+\theta(x)-\theta(\bar{x})}{d\left(x, X^{*}\right)}: x \in X \backslash X^{*}\right\}=\inf \left\{\bar{x}_{1}+x_{2}\right\}=\bar{x}_{1}>0 .
$$

Hence, without checking the definition of the weak sharpness as in Example 3.4, by applying Corollary 3.1 , we can also claim that $X^{*}$ is weakly sharp.

Following the proof of Theorem 3.1, we have the following result. This can be also seen as new characterizations of weak sharpness of solution sets for MVIs in the sense of Huang and He [16].

Corollary 3.2. The following statements are equivalent:

(a) There exists $\alpha>0$ such that (3.6) holds.

(b) There exists $\alpha>0$ such that for each $x \in X$

$$
\alpha d\left(x, X^{*}\right) \leq\left\langle F\left(P_{X^{*}}(x)\right), x-P_{X^{*}}(x)\right\rangle+\theta(x)-\theta\left(P_{X^{*}}(x)\right) .
$$

(c) There exists $\alpha>0$ such that for any $x^{*} \in X^{*}$ and $v \in T_{X}\left(x^{*}\right) \cap N_{X^{*}}\left(x^{*}\right)$

$$
\left\langle F\left(x^{*}\right), v\right\rangle+\theta^{\prime}\left(x^{*}, v\right) \geq \alpha\|v\| .
$$

Remark 3.3. Requiring that the function $F$ is continuous and pseudomonotone ${ }^{+}$, Al-Homidan, Ansari and Nguyen [3, Theorem 1] proved that the solution set $X^{*}$ of variational inequality (2.2) is weakly sharp if and ly if there is a positive number $\alpha$ such that

$$
\left\langle F\left(P_{X^{*}}(x)\right), x-P_{X^{*}}(x)\right\rangle \geq \alpha d\left(x, X^{*}\right) \text { for all } x \in X .
$$

Noticing that the pseudomonotonicity ${ }^{+}$of $F$ implies that $F$ is constant on $X^{*}$ (see [24, Proposition 2] and the proof of [3, Theorem 1] is relied on the continuity of $F$. Hence, Corollary 3.2 improves [3, Theorem 1] by relaxing conditions on $F$.

We can also relax the continuity of the function $F$ improving [23, Theorem 3.2].

Corollary 3.3. Assume that $F$ is a constant on $X^{*}$ and let $x^{*} \in X^{*}$. If there exists $\alpha>0$ such that

$$
\alpha d\left(x, X^{*}\right) \leq\left\langle F\left(x^{*}\right), x-x^{*}\right\rangle+\theta(x)-\theta\left(x^{*}\right) \quad \text { for all } x \in X,
$$

then $X^{*}$ is weakly sharp. 
Proof. For all $y^{*}, z^{*} \in X^{*}$, we have

$$
\left\langle F\left(z^{*}\right), y^{*}-z^{*}\right\rangle+\theta\left(y^{*}\right)-\theta\left(z^{*}\right) \geq 0,
$$

and

$$
\left\langle F\left(y^{*}\right), z^{*}-y^{*}\right\rangle+\theta\left(z^{*}\right)-\theta\left(y^{*}\right) \geq 0 .
$$

Since $F$ is constant on $X^{*}, F\left(y^{*}\right)=F\left(z^{*}\right)$. Hence

$$
\left\langle F\left(z^{*}\right), y^{*}-z^{*}\right\rangle+\theta\left(y^{*}\right)-\theta\left(z^{*}\right)=0 .
$$

By the constancy of $F$ on $X^{*}$, for all $x \in X$, we have

$$
\begin{aligned}
& \left\langle F\left(P_{X^{*}}(x)\right), x-P_{X^{*}}(x)\right\rangle+\theta(x)-\theta\left(P_{X^{*}}(x)\right) \\
= & \left\langle F\left(P_{X^{*}}(x)\right), x-x^{*}\right\rangle+\theta(x)-\theta\left(x^{*}\right) \\
+ & \left\langle F\left(P_{X^{*}}(x)\right), x^{*}-P_{X^{*}}(x)\right\rangle+\theta\left(x^{*}\right)-\theta\left(P_{X^{*}}(x)\right) \\
= & \left\langle F\left(x^{*}\right), x-x^{*}\right\rangle+\theta(x)-\theta\left(x^{*}\right) \geq \alpha d\left(x, X^{*}\right) .
\end{aligned}
$$

Thus, by Corollary 3.2 and Remark 3.2, $X^{*}$ is weakly sharp.

We next present results on the finite convergence of sequences generated by an inexact proximal point algorithm for solving (2.1) under weak sharpness of the solution set.

Let $\left\{\lambda_{k}\right\} \subset(0, \infty)$ and $\left\{e_{k}\right\} \subset H$. We, starting from an arbitrary point $x_{1} \in H$, generate an iteration sequence $\left\{x_{k}\right\}$ in accordance with the rule: for $k=1,2, \cdots$

$$
x_{k+1} \in X_{k+1}:=\left\{y \in X:\left\langle F(y)+\lambda_{k}^{-1}\left(y-x_{k}+e_{k}\right), x-y\right\rangle+\theta(x)-\theta(y) \geq 0, \forall x \in X\right\}
$$

We note that if $F$ is monotone on $X$, then $X_{k}$ is always nonempty for all $k=2,3, \cdots$

Theorem 3.2. Let $X^{*}$ be weakly sharp, $X_{k} \neq \emptyset$ for $k=2,3, \cdots$ and $\left\{x_{k}\right\}$ be a sequence generated by inexact proximal point method (3.15) with $\liminf _{k \rightarrow \infty} \lambda_{k}>0$. Assume that

(a) The sequence $\left\{x_{k+1}-x_{k}\right\}$ converges strongly to 0 ;

(b) The sequence $\left\{e_{k}\right\}$ converges strongly to 0 .

Then $x_{k} \in X^{*}$ for all $k$ large enough if, in addition, one of the following conditions holds:

(i) $F$ is monotone on $X$ and constant on $X^{*}$;

(ii) $H$ is finite dimensional, $F$ is monotone on $X$ and continuous on $X^{*}$ and $\left\{P_{X^{*}}\left(x_{k}\right)\right\}$ is bounded;

(iii) $F$ is monotone on $X$ and continuous on $X^{*}$ and $\left\{P_{X^{*}}\left(x_{k}\right)\right\}$ converges strongly to some $\bar{x} \in X^{*}$;

(iv) $H$ is finite dimensional, $F$ is continuous on $X^{*},\left\{x_{k}\right\}$ is bounded and all accumulation points belong to $X^{*}$;

(v) $F$ is continuous on $X^{*}$ and $\left\{x_{k}\right\}$ converges strongly to some $\bar{y} \in X^{*}$;

(vi) $F$ is uniformly continuous on $X$ and constant on $X^{*}$ and $\left\{d\left(x_{k}, X^{*}\right)\right\}$ converges to 0 ;

Proof. From (3.15), we find, for all $x \in X$, that

$$
\begin{aligned}
& \left\langle F\left(x_{k+1}\right), x_{k+1}-x\right\rangle+\theta\left(x_{k+1}\right)-\theta(x) \\
\leq & \frac{1}{\lambda_{k}}\left(\left\langle x_{k+1}-x_{k}, x-x_{k+1}\right\rangle+\left\langle e_{k}, x-x_{k+1}\right\rangle\right) \\
\leq & \frac{1}{\lambda_{k}}\left(\left\|x_{k+1}-x_{k}\right\| \cdot\left\|x-x_{k+1}\right\|+\left\|e_{k}\right\| \cdot\left\|x-x_{k+1}\right\|\right) .
\end{aligned}
$$


Assume that the conclusion is not true. Then there exists a subsequence $\left\{x_{k_{i}}\right\}$ of $\left\{x_{k}\right\}$ such that $x_{k_{i}} \notin X^{*}$ for all $i$. For each $i$, set $y_{k_{i}}=P_{X^{*}}\left(x_{k_{i}}\right)$. It follows that $\left\|x_{k_{i}}-y_{k_{i}}\right\|=d\left(x_{k_{i}}, X^{*}\right)>0$ for all $i$.

(i) Since $F$ is a constant on $X^{*}$, we find from Corollary 3.2 that there exists $\alpha>0$ such that

$$
\alpha|| y_{k_{i}}-x_{k_{i}}||=\alpha d\left(x_{k_{i}}, X^{*}\right) \leq\left\langle F\left(y_{k_{i}}\right), x_{k_{i}}-y_{k_{i}}\right\rangle+\theta\left(x_{k_{i}}\right)-\theta\left(y_{k_{i}}\right) .
$$

Using the monotonicity of $F$, we have

$$
\alpha\left\|x_{k_{i}}-y_{k_{i}}\right\| \leq\left\langle F\left(x_{k_{i}}\right), x_{k_{i}}-y_{k_{i}}\right\rangle+\theta\left(x_{k_{i}}\right)-\theta\left(y_{k_{i}}\right) .
$$

Taking $k:=k_{i}$ and $x=y_{k_{i}+1}$ in (3.16) and using (3.18), one has

$$
\alpha|| x_{k_{i}+1}-y_{k_{i}+1} \| \leq \frac{1}{\lambda_{k_{i}}}\left(\left\|x_{k_{i}+1}-x_{k_{i}}\right\| \cdot\left\|y_{k_{i}+1}-x_{k_{i}+1}\right\|+\left\|e_{k_{i}}\right\| \cdot\left\|y_{k_{i}+1}-x_{k_{i}+1}\right\|\right) .
$$

Dividing both sides of the latter inequality by $\left\|y_{k_{i}+1}-x_{k_{i}+1}\right\|>0$, we get

$$
\alpha \leq \frac{1}{\lambda_{k_{i}}}\left(\left\|x_{k_{i}+1}-x_{k_{i}}\right\|+\left\|e_{k_{i}}\right\|\right) .
$$

Since $\liminf _{k \rightarrow \infty} \lambda_{k}>0, \lim _{k \rightarrow \infty}\left(x_{k+1}-x_{k}\right)=0, \lim _{k \rightarrow \infty} e_{k}=0$, it follows from the latter inequality that $\alpha \leq 0$. This contradicts $\alpha>0$. Thus, $x_{k} \in X^{*}$ for sufficiently large $k$.

(ii) (iii) In both cases, we may assume that $\left\{y_{k_{i}}\right\}$ converges strongly to some $\bar{x} \in X^{*}$. By Theorem 3.1, there exists $\alpha>0$ such that

$$
\alpha|| x_{k_{i}+1}-y_{k_{i}+1} \| \leq\left\langle F(\bar{x}), x_{k_{i}+1}-y_{k_{i}+1}\right\rangle+\theta\left(x_{k_{i}+1}\right)-\theta\left(y_{k_{i}+1}\right) .
$$

Using the monotonicity of $F$ and (3.16), one has

$$
\begin{aligned}
\alpha\left\|x_{k_{i}+1}-y_{k_{i}+1}\right\| \leq & \left.F\left(x_{k_{i}+1}\right), x_{k_{i}+1}-y_{k_{i}+1}\right\rangle+\theta\left(x_{k_{i}+1}\right)-\theta\left(y_{k_{i}+1}\right) \\
& +\left\langle F\left(y_{k_{i}+1}\right)-F\left(x_{k_{i}+1}\right), x_{k_{i}+1}-y_{k_{i}+1}\right\rangle \\
& +\left\langle F(\bar{x})-F\left(y_{k_{i}+1}\right), x_{k_{i}+1}-y_{k_{i}+1}\right\rangle \\
\leq & \frac{1}{\lambda_{k_{i}}}\left(\left\|x_{k_{i}+1}-x_{k_{i}}\right\| \cdot\left\|y_{k_{i}+1}-x_{k_{i}+1}\right\|+\left\|e_{k_{i}}\right\| \cdot\left\|y_{k_{i}+1}-x_{k_{i}+1}\right\|\right) \\
& +\left\|F\left(y_{k_{i}+1}\right)-F(\bar{x})\right\| \cdot\left\|x_{k_{i}+1}-y_{k_{i}+1}\right\| .
\end{aligned}
$$

Dividing both sides by $\left\|x_{k_{i}+1}-y_{k_{i}+1}\right\|>0$, we get

$$
\alpha \leq \frac{1}{\lambda_{k_{i}}}\left(\left\|x_{k_{i}+1}-x_{k_{i}}\right\|+\left\|e_{k_{i}}\right\|\right)+\left\|F\left(y_{k_{i}+1}\right)-F(\bar{x})\right\| .
$$

This yields $\alpha \leq 0$, which is a contradiction. Therefore, $x_{k} \in X^{*}$ for all $k$ sufficiently large.

(iv) (v) In both cases, we may assume that $\left\{x_{k_{i}}\right\}$ converges strongly to $\bar{y} \in X^{*}$. By Theorem 3.1, there exists $\alpha>0$ such that

$$
\alpha|| x_{k_{i}+1}-y_{k_{i}+1} \| \leq\left\langle F(\bar{y}), x_{k_{i}+1}-y_{k_{i}+1}\right\rangle+\theta\left(x_{k_{i}+1}\right)-\theta\left(y_{k_{i}+1}\right) .
$$

Using (3.16), we have

$$
\begin{aligned}
\alpha\left\|x_{k_{i}+1}-y_{k_{i}+1}\right\| \leq & \left.F\left(x_{k_{i}+1}\right), x_{k_{i}+1}-y_{k_{i}+1}\right\rangle+\theta\left(x_{k_{i}+1}\right)-\theta\left(y_{k_{i}+1}\right) \\
& +\left\langle F(\bar{y})-F\left(x_{k_{i}+1}\right), x_{k_{i}+1}-y_{k_{i}+1}\right\rangle \\
\leq & \frac{1}{\lambda_{k_{i}}}\left(\left\|x_{k_{i}+1}-x_{k_{i}}\right\| \cdot\left\|y_{k_{i}+1}-x_{k_{i}+1}\right\|+\left\|e_{k_{i}}\right\| \cdot\left\|y_{k_{i}+1}-x_{k_{i}+1}\right\|\right) \\
& +\left\|F\left(x_{k_{i}+1}\right)-F(\bar{y})\right\| \cdot\left\|x_{k_{i}+1}-y_{k_{i}+1}\right\| .
\end{aligned}
$$


It follows that

$$
\alpha \leq \frac{1}{\lambda_{k_{i}}}\left(\left\|x_{k_{i}+1}-x_{k_{i}}\right\|+\left\|e_{k_{i}}\right\|\right)+\left\|F\left(x_{k_{i}+1}\right)-F(\bar{y})\right\| .
$$

This implies $\alpha \leq 0$, which contradicts $\alpha>0$. Therefore, $x_{k} \in X^{*}$ for all $k$ sufficiently large.

(vi) Since $F$ is a constant on $X^{*}$, we find from Corollary 3.2 that there exists $\alpha>0$ such that

$$
\alpha\left\|y_{k_{i}}-x_{k_{i}}\right\| \leq\left\langle F\left(y_{k_{i}}\right), x_{k_{i}}-y_{k_{i}}\right\rangle+\theta\left(x_{k_{i}}\right)-\theta\left(y_{k_{i}}\right) .
$$

By (3.16), one has

$$
\begin{aligned}
\alpha\left\|y_{k_{i}+1}-x_{k_{i}+1}\right\| \leq & \left\langle F\left(x_{k_{i}+1}\right), x_{k_{i}+1}-y_{k_{i}+1}\right\rangle+\theta\left(x_{k_{i}+1}\right)-\theta\left(y_{k_{i}+1}\right) \\
& +\left\langle F\left(y_{k_{i}+1}-F\left(x_{k_{i}+1}\right), x_{k_{i}+1}-y_{k_{i}+1}\right\rangle\right. \\
\leq & \frac{1}{\lambda_{k_{i}}}\left(\left\|x_{k_{i}+1}-x_{k_{i}}\right\| \cdot\left\|y_{k_{i}+1}-x_{k_{i}+1}\right\|+\left\|e_{k_{i}}\right\| \cdot\left\|y_{k_{i}+1}-x_{k_{i}+1}\right\|\right) \\
& +\| F\left(y_{k_{i}+1}-F\left(x_{k_{i}+1}\right)\|\cdot\| x_{k_{i}+1}-y_{k_{i}+1} \| .\right.
\end{aligned}
$$

Since $F$ is uniformly continuous on $X$, there exists $\delta>0$ such that

$$
\|F(x)-F(y)\|<\frac{\alpha}{4}, \quad \text { for all } x, y \in X \quad \text { with } \quad\|x-y\|<\delta .
$$

Moreover, since $\lim _{i \rightarrow \infty}|| x_{k_{i}}-y_{k_{i}} \mid=\lim _{i \rightarrow \infty} d\left(x_{k_{i}}, X^{*}\right)=0$, we find that there exists $i_{1} \in \mathbb{N}$ such that ||$x_{k_{i}}-y_{k_{i}}||<\delta$ for all $i \geq i_{1}$. Thus,

$$
\| F\left(y_{k_{i}+1}-F\left(x_{k_{i}+1}\right) \|<\frac{\alpha}{4}, \quad \forall i \geq i_{1} .\right.
$$

Since $\liminf _{k \rightarrow \infty} \lambda_{k}>0$, there exist $q>0$ and $i_{2} \in \mathbb{N}$ such that $\lambda_{k_{i}} \geq q$ for all $i \geq i_{2}$. By (a) and (b), there exists $i_{0} \geq \max \left\{i_{1}, i_{2}\right\}$ such that

$$
\left\|x_{k_{i}+1}-x_{k_{i}}\right\|<\frac{q \alpha}{4}, \quad \text { and } \quad\left\|e_{k_{i}}\right\|<\frac{q \alpha}{4}, \quad \forall i \geq i_{0} .
$$

It follows from (3.19) that

$$
\begin{aligned}
\alpha & \leq \frac{1}{\lambda_{k_{i}}}\left(\left\|x_{k_{i}+1}-x_{k_{i}}\right\|+\left\|e_{k_{i}}\right\|\right)+\| F\left(y_{k_{i}+1}-F\left(x_{k_{i}+1}\right) \|\right. \\
& \leq \frac{1}{q}\left(\frac{q \alpha}{4}+\frac{q \alpha}{4}\right)+\frac{\alpha}{4}=\frac{3 \alpha}{4}, \quad \forall i \geq i_{0} .
\end{aligned}
$$

This contradicts $\alpha>0$. Therefore, $x_{k} \in X^{*}$ for all $k$ sufficiently large.

Remark 3.4. Theorem 3.1 in [27] is a special case of Theorem 3.2 (iii). Hence, Theorem 3.2 generalizes several results in the literature (see, [27, Remark 3.5]). In our result, we also consider some cases that mapping $F$ is not monotone. This is new even for the variational inequality setting.

To conclude this paper, we consider an exact proximal point method. In (3.15), we take $e_{k}=0$ for all $k$. For $x_{1} \in H$, let $\left\{x_{k}\right\}$ be the sequence by

$$
\left\langle F\left(x_{k+1}\right)+\lambda_{k}^{-1}\left(x_{k+1}-x_{k}\right), x-x_{k+1}\right\rangle+\theta(x)-\theta\left(x_{k+1}\right) \geq 0, \quad \forall x \in X .
$$

We recall following lemma which will be used in the proof of our result (see, e.g., [5]). 
Lemma 3.1. Let $D$ be a nonempty subset of $H$ and let $\left\{x_{k}\right\}$ be a sequence in $H$. Assume that $\left\{x_{k}\right\}$ in $H$ is Fejér monotone with respect to D, that is,

$$
\left\|x_{k+1}-x\right\| \leq\left\|x_{k}-x\right\|,
$$

for all $k \in \mathbb{N}$ and $x \in D$. Then $\left\{P_{D}\left(x_{k}\right)\right\}$ converges strongly to a point in $D$.

Corollary 3.4. Let $F$ be monotone on $X$ and continuous on $X^{*}$ and let $X^{*}$ be weakly sharp. Let $\left\{x_{k}\right\}$ be a sequence generated in (3.20) with $\liminf _{k \rightarrow \infty} \lambda_{k}>0$. Then, $x_{k} \in X^{*}$ for all $k$ sufficiently large.

Proof. We can rewrite (3.20) as

$$
\left\langle F\left(x_{k+1}\right), x-x_{k+1}\right\rangle+\theta(x)-\theta\left(x_{k+1}\right)+\frac{1}{2 \lambda_{k}}\left(\left\|x_{k}-x\right\|^{2}-\left\|x_{k+1}-x\right\|^{2}-\left\|x_{k+1}-x_{k}\right\|^{2}\right) \geq 0,
$$

for all $x \in X$. Let $x^{*}$ be a solution of (2.1). Then

$$
\left\langle F\left(x^{*}\right), x_{k+1}-x^{*}\right\rangle+\theta\left(x_{k+1}\right)-\theta\left(x^{*}\right) \geq 0 .
$$

By the monotonicity of $F$, we have

$$
\left\langle F\left(x_{k+1}\right), x^{*}-x_{k+1}\right\rangle+\theta\left(x^{*}\right)-\theta\left(x_{k+1}\right) \leq\left\langle F\left(x^{*}\right), x^{*}-x_{k+1}\right\rangle+\theta\left(x^{*}\right)-\theta\left(x_{k+1}\right) \leq 0 .
$$

Hence, it follows from (3.21) that

$$
\left\|x_{k+1}-x^{*}\right\|^{2} \leq\left\|x_{k}-x^{*}\right\|^{2}-\left\|x_{k+1}-x_{k}\right\|^{2} .
$$

This implies that $\left\{x_{k}\right\}$ is Fejér monotone with respect to $X^{*}, \lim _{k \rightarrow \infty}\left\|x_{k}-x^{*}\right\|$ exists and $\lim _{k \rightarrow \infty} \| x_{k+1}-$ $x_{k} \|=0$. Using Lemma 3.1, we find that $\left\{P_{X^{*}}\left(x_{k}\right)\right\}$ converges strongly to some $\bar{x} \in X^{*}$. Applying Theorem 3.2, we obtain that $x_{k} \in X^{*}$ for all $k$ large enough.

\section{Acknowledgments}

This article was supported by the National Natural Science Foundation of China under Grant No.11401152. The first author was also supported by the Research Fund for International Young Scientists under Grant No. 1181101157 and the China Postdoctoral Science Foundation under Grant No. 2017M6200421.

\section{REFERENCES}

[1] J.-P. Aubin, I. Ekeland, Applied Nonlinear Analysis, Wiley-Interscience, Mineola, 1984.

[2] S. Al-Homidan, Q.H. Ansari, S. Burachik, Weak sharp solutions for generalized variational inequalities, Positivity, 21 (2017), 1067-1088.

[3] S. Al-Homidan, Q.H. Ansari, L.V. Nguyen, Finite convergence analysis and weak sharp solutions for variational inequalities, Optim. Lett. 11 (2017), 1647-1662.

[4] S. Al-Homidan, Q.H. Ansari, L.V. Nguyen, Weak sharp solutions for nonsmooth variational inequalities, J. Optim. Theory Appl. 175 (2017), 683-701.

[5] H.H. Bauschke, P.L. Combettes, Convex Analysis and Monotone Operator Theory in Hilbert Spaces, Springer, New York, 2011.

[6] F.E. Browder, On the unification of the calculus of variations and the theory of monotone nonlinear operators in Banach spaces, Proc. Nat. Acad. Sci. USA 56 (1966), 419-425.

[7] J.V. Burke, M.C. Ferris, Weak sharp minima in mathematical programming, SIAM J. Control Optim. 31 (1993), 13401359.

[8] J.V. Burke, S. Deng, Weak sharp minima revisited, Part III: error bounds for differentiable convex inclusions, Math. Program. 116 (2009), 37-56. 
[9] J.V. Burke, S. Deng, Weak sharp minima revisited, part II: application to linear regularity and error bounds, Math. Program. 104 (2005), 235-261.

[10] J.V. Burke, S. Deng, Weak sharp minima revisited, part I: Basic theory, Control and Cybernetics 31 (2002), $439-469$.

[11] C. Chen, S. Ma, J. Yang, A general inertial proximal point algorithm for mixed variational inequality problem, SIAM J. Optim. 25 (2015), 2120-2142.

[12] L. Cromme, Strong uniqueness, Numer. Math. 29 (1978), 179-193.

[13] M.C. Ferris, Weak Sharp Minima and Penalty Functions in Mathematical Programming, Ph.D. Thesis, University of Cambridge, Cambridge, 1988.

[14] M.C. Ferris, Finite termination of the proximal point algorithm, Math. Program. 50 (1991), 359-366.

[15] D. Goeleven, Existence and uniqueness for a linear mixed variational inequality arising in electrical circuits with transistors, J. Optim. Theory Appl. 138 (2008), 397-406.

[16] H. Huang, M. He, Weak sharp solution of mixed variational inequalities in Banach spaces, Optim. Lett. 12 (2018), 287299.

[17] Y. Hua, W. Song, Weak sharp solutions for variational inequalities in Banach spaces, J. Math. Anal. Appl. 374 (2011), 118-132.

[18] A. Jourani, Hoffman's error bounds, local controllability, and sensitivity analysis, SIAM J. Control Optim. 38 (2000), 947-970.

[19] A. Jayswal, S. Singh, Characterization of weakly sharp solutions of a variational-type inequality with convex functional, Ann. Oper. Res. 269 (2018), 297-315.

[20] I.V. Konnov, Application of the proximal point method to nonmonotone equilibrium problems, J. Optim. Theory Appl. 119 (2003), 317-333.

[21] I.V. Konnov, E.O. Volotskaya, Mixed variational inequalities and economic equilibrium problems, J. Appl. Math. 2 (2002), 289-314.

[22] C. Lescarret, Cas d'addition des applications monotones maximales dans un espace de Hilbert, C. R. Acad. Sci. Paris 261 (1965), 1160-1163.

[23] Y. Liu, Weakly sharp solutions and finite convergence of algorithms for a variational inequality problem, Optimization, 67 (2018), 329-340.

[24] Y. Liu, Z. Wu, Characterization of weakly sharp solutions of a variational inequality by its primal gap function, Optim. Lett. 10 (2016), 563-576.

[25] P. Marcotte, D.L. Zhu, Weak sharp solutions of variational inequalities, SIAM J. Optim. 9 (1999), 179-189.

[26] S. Matsushita, L. Xu, Finite convergence of the proximal point algorithm for variational inequality problems, Set-Valued Var. Anal. 21 (2013), 297-309.

[27] S. Matsushita, L. Xu, On Finite Convergence of Iterative Methods for Variational Inequalities in Hilbert Spaces, J. Optim. Theory Appl. 161 (2014), 701-715.

[28] M. Patriksson, Unified framework of descent algorithms for nonlinear programs and variational inequalities [PhD thesis], Linkoping, Sweden: Department of Mathematics, Linkoping Institute of Technology, 1993.

[29] V.I. Opoitsev, Nonlinear Systemostatics, vol. 31, Nauka, Moscow, 1986.

[30] B.T. Polyak, Sharp minima, Institute of Control Sciences Lecture Notes, Moscow, USSR, 1979; Presented at the IIASA Workshop on Generalized Lagrangians and Their Applications, IIASA, Laxenburg, Austria, 1979.

[31] J. Xiong, J. Li, Weak sharpness for set-valued variational inequalities and applications to finite termination of iterative algorithms, Optimization, 65 (2016), 1585-1597.

[32] N.H. Xiu, J.Z. Zhang, On finite convergence of proximal point algorithms for variational inequalities, J. Math. Anal. Appl. 312 (2005), 148-158.

[33] Z. Wu, S.Y. Wu, Weak sharp solutions of variational inequalities in Hilbert spaces, SIAM J. Optim. 14 (2004), $1011-1027$.

[34] Z. Wu, Characterizations of weakly sharp solutions for a variational inequality with a pseudomonotone mapping, Eur. J. Oper. Res. 265 (2018), 448-453. 